\title{
Baryon-dark matter coincidence in mirrored unification
}

\author{
Masahiro Ibe, ${ }^{1,2}$ Ayuki Kamada $\odot,{ }^{3}$ Shin Kobayashi $\odot,{ }^{1,2}$ Takumi Kuwahara $\odot,{ }^{3}$ and Wakutaka Nakano ${ }^{1,2}$ \\ ${ }^{1}$ Kavli IPMU (WPI), UTIAS, The University of Tokyo, Kashiwa, Chiba 277-8583, Japan \\ ${ }^{2}$ ICRR, The University of Tokyo, Kashiwa, Chiba 277-8582, Japan \\ ${ }^{3}$ Center for Theoretical Physics of the Universe, Institute for Basic Science (IBS), Daejeon 34126, Korea
}

(Received 24 July 2019; published 18 October 2019)

\begin{abstract}
About $80 \%$ of the mass of the present Universe is made up of the unknown (dark matter), while the rest is made up of ordinary matter. It is a very intriguing question why the mass densities of dark matter and ordinary matter (mainly baryons) are close to each other. It may hint the identity of dark matter and furthermore the structure of a dark sector. A mirrored world provides a natural explanation to this puzzle. On the other hand, if the mirror-symmetry breaking scale is low, it tends to cause cosmological problems. In this work, we propose a mirrored unification framework, which breaks mirror symmetry at the grand unified scale, but still addresses the puzzle. The dark matter mass is strongly related with the dynamical scale of quantum chromodynamics, which explains the closeness of the dark matter and baryon masses. Intermediate-energy portal interactions share the generated asymmetry between the visible and dark sectors. Furthermore, our framework is safe from cosmological issues by providing low-energy portal interactions to release the superfluous entropy of the dark sector into the visible sector.
\end{abstract}

DOI: $10.1103 /$ PhysRevD.100.075022

\section{INTRODUCTION}

Cosmological observations have established that the mass of the present Universe is made up of so-called dark matter (DM) in addition to ordinary matter. The mass density of DM is about five times larger than that of ordinary matter, i.e., standard model (SM) baryons [1]. The observed closeness of the mass densities may be a hint on DM and dark sector physics. If DM (dark sector) has nothing to do with the SM baryon (visible sector), it is puzzling why their mass densities are close to each other.

The concept of a mirror world is a natural option to explain this puzzle (see Refs. [2-8] for earlier works). In recent years, the mirror world scenarios combined with twin Higgs models also attract attention since they ameliorate the naturalness problem [9-12]. In those scenarios, the dark sector contains mirror partners of the SM particles, and therefore the coincidence is naturally realized. However, if mirror $\mathbb{Z}_{2}$ symmetry is kept at a low-energy scale, mirrorworld models tend to be inconsistent with cosmology because the dark sector inevitably includes light particles such as the mirror partners of neutrinos and photon.

Instead, in this work, we pursue a mirrored grand unified theory (GUT) framework, in which $\mathbb{Z}_{2}$ symmetry is

Published by the American Physical Society under the terms of the Creative Commons Attribution 4.0 International license. Further distribution of this work must maintain attribution to the author(s) and the published article's title, journal citation, and DOI. Funded by SCOAP ${ }^{3}$. broken at a GUT scale. We consider a GUT model with gauge dynamics of $G_{\mathrm{VGUT}} \times G_{\mathrm{DGUT}}$ (with a gauge group $G=G_{\mathrm{VGUT}}=G_{\mathrm{DGUT}}$ ) and an exchanging symmetry between $G_{\mathrm{VGUT}}$ and $G_{\mathrm{DGUT}}$. References $[13,14]$ are in a similar line but with a focus on the coincidence of the dynamical scales [13] and with low-energy $\mathbb{Z}_{2}$ breaking [14]. It is remarkable that $\mathbb{Z}_{2}$-symmetry breaking at a highenergy scale does not lose good features as long as the lightest dark baryons are DM [15-54] (see Ref. [55] for a review). The baryon-DM coincidence puzzle is divided into two subproblems: the coincidence of masses and that of number densities between baryons and DM. As for the mass coincidence, the key ingredient is the correspondence between dynamical scales of each sector: the baryon and DM masses are determined by them. Such a correspondence can be achieved once the gauge couplings are related with each other at the GUT scale. As we see, $\mathbb{Z}_{2}$-symmetry breaking below the GUT scale does not spoil this correspondence.

As for the number density, we consider the asymmetric dark matter (ADM) framework [56-66] (see also Refs. [67-69] for reviews). Since the dark baryons have an annihilation cross section as large as the SM baryons have $[70,71]$, the number density of DM is dominated by an asymmetry between particle and antiparticle. The asymmetries in the two sectors are equilibrated when a portal interaction is efficient at an intermediate scale.

After decoupling of the portal interaction, the entropy densities in the two sectors are conserved separately: the excessive entropy in the dark sector gives a significant 
contribution to the dark radiation [72]. Therefore, two types of portal interactions are needed for viable (composite) ADM scenarios: intermediate-energy portal interactions to share the asymmetry, and low-energy portal interactions to release the superfluous entropy of the dark sector into the visible sector. Our framework indeed provides such portal interactions, and thus explains the baryon-DM coincidence puzzle in a self-contained manner.

Our framework is based on a supersymmetric grand unified theory (SUSY GUT), in which the $\mathbb{Z}_{2}$ symmetry is manifest above the GUT scale. The gauge structure of each sector at low energy depends on a choice of vacuum at the GUT scale. In our framework, the visible sector is reduced to the SM, while the dark sector follows two-step symmetry breaking and then has a dynamics similar to quantum chromodynamics (QCD) and quantum electrodynamics (QED). The second symmetry breaking in the dark sector provides the intermediate-energy portal interactions and tiny kinetic mixing of visible photon and dark photon. SUSY plays key roles to achieve gauge coupling unification in the visible sector and to ensure the existence of degenerate vacua with various breaking patterns of the GUT gauge group. The degenerate vacua permit the development of the dark sector in a different way from our sector. This feature allows us to have the gauge dynamics in the dark sector that is different from the one in the SM at the low-energy scale. With the variety of the unbroken gauge groups, the dark sector and the visible sector have different phenomenology, while the dynamical scales in the two sectors are correlated due to the mirror symmetric gauge group at the GUT scale. Electroweak symmetry breaking in the visible sector and dark QED breaking are triggered by SUSY breaking effects.

The organization of this article is as follows: we demonstrate our framework with the use of a concrete example based on a $G=S U(5)$ gauge dynamics in the next section. We then discuss a symmetry breaking pattern in the dark sector. In particular, we choose a pattern to realize the minimal model that provides key ingredients for the composite ADM scenarios. Next, we explain how the effective theory at an intermediate scale and below works in the context of the composite ADM, which is based on our previous works $[50,73]$. We discuss the coincidence of confinement scales in the concrete model. The last section is devoted to concluding remarks of this article.

\section{MIRRORED UNIFICATION MODEL}

We consider a concrete model with $G=S U(5)$ to demonstrate our framework. $\mathbb{Z}_{2}$ is the symmetry interchanging $S U(5)_{\text {VGUT }}$ and $S U(5)_{\text {DGUT }}$. Under the $\mathbb{Z}_{2}$ symmetry, dimensionless couplings in the two sectors are identified, while the mass parameters softly break the $\mathbb{Z}_{2}$ symmetry.

We show the particle contents of the model in Table I, which are similar to those of the minimal SUSY $S U(5)$ GUT in each sector. The chiral multiplets, $\Psi_{V}$ and $\Phi_{V}$, contain all
TABLE I. Matter and Higgs contents in the $S U(5)_{\mathrm{VGUT}} \times$ $S U(5)_{\text {DGUT }}$ model. The subscript $S=V, D$ represents the sectors: the fields are charged under $S U(5)_{\mathrm{VGUT}}$ for $S=V$ and charged under $S U(5)_{\text {DGUT }}$ for $S=D . i=1,2,3$ denotes the generations of matter chiral multiplets in each sector.

\begin{tabular}{lcc}
\hline \hline & $S U(5)_{\text {VGUT,DGUT }}$ & $U(1)_{X}$ \\
\hline$\Psi_{S i}$ & $\mathbf{1 0}$ & 1 \\
$\Phi_{S i}$ & $\overline{\mathbf{5}}$ & -3 \\
$\bar{N}_{i}, \bar{N}_{i}^{\prime}$ & $\mathbf{1}$ & 5 \\
$H_{S}$ & $\mathbf{5}$ & -2 \\
$\bar{H}_{S}$ & $\overline{\mathbf{5}}$ & 2 \\
$X_{S}$ & $\mathbf{5}$ & -2 \\
$\bar{X}_{S}$ & $\overline{\mathbf{5}}$ & 2 \\
$\Sigma_{S}$ & $\mathbf{2 4}$ & 0 \\
\hline \hline
\end{tabular}

the SM fermions. $\Psi_{D}$ and $\Phi_{D}$ include the dark quarks that provide the ingredients of the composite DM. $\bar{N}_{i}$ and $\bar{N}_{i}^{\prime}$ are the right-handed neutrinos, which are doublets under the $\mathbb{Z}_{2}$ symmetry. $U(1)_{X}$ denotes a global $B-L$ symmetry compatible with the unified gauge group. It should be noted that the model has extra Higgs quintuplets, $\left(X_{S}, \bar{X}_{S}\right)$, in addition to the usual Higgs quintuplets, $\left(H_{S}, \bar{H}_{S}\right)$.

Once $B-L$ asymmetry is generated, it is shared with the dark sector via the $B-L$ portal operators, which we show later. Since the $B-L$ number is approximately conserved in two sectors, the global symmetry ensures the stability of DM and the absence of operators that wash out the generated asymmetry. We assume that the $B-L$ asymmetry is generated via standard thermal leptogenesis [74] (see Refs. [75-77] for reviews) as for the concrete model. The DM and baryon abundances relate to parameters in the neutrino sector in this model.

The minimal $S U(5)$ GUT model with $\mathcal{O}(1)$ TeV sfermions contradicts the nucleon decay experiments [78-81]. To avoid rapid nucleon decay, we simply assume a split spectrum for sparticles [82-85], where sfermions and gravitinos have masses of $\mathcal{O}\left(10^{2}\right) \mathrm{TeV}$ while the masses of the gauginos and the Higgsinos are of $\mathcal{O}(100) \mathrm{GeV}-\mathcal{O}(1) \mathrm{TeV}$.

Since the lightest supersymmetric particle (LSP) is stable, it contributes to the DM abundance. We assume that its fraction to the total DM abundance is subdominant. Such a situation can be easily achieved when the Higgsinos are the LSPs in each sector. Here, the Higgsinos are the fermionic partners of the Higgs fields breaking the electroweak symmetry in the visible sector and the dark QED, respectively. We assume that the dark Higgsino is lighter than the Higgsino in the visible sector because the dark Higgsino is less constrained than the visible Higgsino. ${ }^{1}$

\footnotetext{
${ }^{1}$ If the visible Higgsino is lighter than the dark Higgsino, we should take into account the overabundance of the Higgsino through thermal freeze-out and through the gravitino decay. While the Higgsino mass is bounded from above to avoid the overabundance, it is also bounded below by collider searches.
} 
Thanks to the supersymmetric counterpart of the kinetic mixing parameter $\epsilon \simeq 10^{-9}$, in our framework the decay of the visible Higgsino to the dark one occurs fast enough not to cause any cosmological issues [73].

Both sectors are mostly sequestered with each other up to higher-dimensional interactions suppressed by the reduced Planck mass $M_{\mathrm{Pl}}$. The superpotential $W_{S}$ gives the Yukawa couplings, the Higgs masses, and the Higgs couplings to fields with subscripts $S=V, D$,

$$
\begin{aligned}
W_{S}= & \Psi_{S} Y_{u} \Psi_{S} H_{S}+\Psi_{S} Y_{d} \Phi_{S} \bar{H}_{S}+H_{S}\left(M_{S}+\lambda \Sigma_{S}\right) \bar{H}_{S} \\
& +\mu_{S} \operatorname{tr}\left(\Sigma_{S}^{2}\right)+\lambda_{\Sigma} \operatorname{tr}\left(\Sigma_{S}^{3}\right)+M_{S}^{\prime} X_{S} \bar{X}_{S}-\xi \frac{\left(X_{S} \bar{X}_{S}\right)^{2}}{M_{\mathrm{Pl}}} .
\end{aligned}
$$

Here, $\lambda, \lambda_{\Sigma}$, $\xi$, and $3 \times 3$ matrices $Y_{u, d}$ are dimensionless coupling constants, while $M_{S}, M_{S}^{\prime}$, and $\mu_{S}$ are dimensionful parameters. We assume $\lambda, \lambda_{\Sigma}$, and $\xi$ are of $\mathcal{O}(1)$ in the following. The $\mathbb{Z}_{2}$ symmetry equates all the dimensionless couplings except the mass parameters in the two sectors: we assume mass hierarchy $M_{D}, M_{D}^{\prime}, \mu_{D} \ll M_{V}, M_{V}^{\prime}, \mu_{V}$.

\section{A. Symmetry breaking patterns}

$S U(5)_{\text {VGUT }}$ is broken down to the gauge group of the SM, $G_{\mathrm{SM}}$, by a vacuum expectation value (VEV) of $\Sigma_{V}$ at the scale of $M_{\mathrm{VGUT}} \simeq \mu_{V}$, while $X$ 's and $H$ 's do not obtain large VEVs. That is, $S U(5)_{\mathrm{VGUT}} \rightarrow G_{\mathrm{SM}}$ is achieved by

$$
\left\langle\Sigma_{V}\right\rangle=\mathcal{O}\left(\mu_{V}\right), \quad\left\langle X_{V} \bar{X}_{V}\right\rangle=0 .
$$

We set $M_{\mathrm{VGUT}}=\mathcal{O}\left(10^{16}\right) \mathrm{GeV}$, which is expected from the unification of extrapolated gauge coupling constants in the supersymmetric SM (SSM).

The vacuum of the dark sector is chosen to be

$$
\left\langle\Sigma_{D}\right\rangle=\mathcal{O}\left(\mu_{D}\right), \quad\left\langle X_{D} \bar{X}_{D}\right\rangle=\mathcal{O}\left(M_{D}^{\prime} M_{\mathrm{Pl}}\right) .
$$

The nonvanishing VEV of $X_{D} \bar{X}_{D}$ is due to the fourth term of Eq. (1). For $\mu_{D} \sim M_{D}^{\prime} \ll M_{\mathrm{Pl}}, S U(5)_{\mathrm{DGUT}}$ is first broken down to $S U(4)_{\mathrm{DGUT}}$ by $\left\langle X_{D}\right\rangle$. $S U(4)_{\mathrm{DGUT}}$ is subsequently broken down to $S U(3)_{D} \times U(1)_{D}$ by $\left\langle\Sigma_{D}\right\rangle$ at $M_{\text {DGUT }}=\mathcal{O}\left(\mu_{D}\right)$. The dark sector results in the model of a composite ADM model in [50,73].

It should be emphasized that the difference between $M_{\mathrm{VGUT}}$ and $M_{\mathrm{DGUT}}$ is advantageous to explain the tiny kinetic mixing between the dark photon and the visible photon [73]. In fact, a higher-dimensional operator,

$$
W_{\mathrm{Pl}}=\frac{c_{\epsilon}}{M_{\mathrm{Pl}}^{2}} \operatorname{tr}\left(\Sigma_{V} \mathcal{W}_{V}\right) \operatorname{tr}\left(\Sigma_{D} \mathcal{W}_{D}\right),
$$

leads to the kinetic mixing parameter of the visible and the dark photons,

$$
\epsilon \simeq \frac{c_{\epsilon} M_{\mathrm{VGUT}} M_{\mathrm{DGUT}}}{M_{\mathrm{Pl}}^{2}} \simeq 10^{-10}\left(\frac{M_{\mathrm{DGUT}}}{10^{10} \mathrm{GeV}}\right) .
$$

We obtain a tiny kinetic mixing parameter $\epsilon=10^{-7}-10^{-10}$ for $M_{\mathrm{DGUT}}=10^{10-13} \mathrm{GeV}$ and $c_{\epsilon}=1$, which satisfies all the constraints including the beam dump experiments [86] and supernova 1987A $[87,88]$ when the dark photon mass is $\mathcal{O}\left(10^{1-2}\right) \mathrm{MeV}$.

\section{B. Intermediate-scale effective theory}

Below $M_{\mathrm{VGUT}}$, we assume the SSM for the visible sector, where a pair of Higgs doublets from $\left(H_{V}, \bar{H}_{V}\right)$ remains almost massless by tuning $M_{V}$ in Eq. (1). All the other components of the extra Higgs have masses of $\mathcal{O}\left(M_{\mathrm{VGUT}}\right)$ in the visible sector.

In the dark sector, $S U(5)_{\mathrm{DGUT}}$ is broken down to $S U(4)_{\mathrm{DGUT}}$ at $\sqrt{M_{\mathrm{DGUT}} M_{\mathrm{Pl}}} \sim 10^{14-16} \mathrm{GeV}$ for $M_{\mathrm{DGUT}}=$ $10^{10-13} \mathrm{GeV}$. The gauge multiplets and the pseudo-Goldstone components of $\left(X_{D}, \bar{X}_{D}\right)$ corresponding to $S U(5)_{\text {DGUT }} /$ $S U(4)_{\text {DGUT }}$ obtain masses of $\mathcal{O}\left(\sqrt{M_{\text {DGUT }} M_{\mathrm{Pl}}}\right) .{ }^{2}$ Below the $S U(5)_{\text {DGUT }}$ breaking scale, the matter and the Higgs multiplets are decomposed into the $S U(4)_{\text {DGUT }}$ multiplets by

$$
\begin{aligned}
& \Psi_{D} \rightarrow A_{D}(\mathbf{6}) \oplus Q_{D}(\mathbf{4}), \quad \Phi_{D} \rightarrow \bar{Q}_{D}(\overline{\mathbf{4}}) \oplus N_{D}(\mathbf{1}), \\
& H_{D} \rightarrow H_{D}(\mathbf{4}) \oplus S_{D}(\mathbf{1}), \quad \bar{H}_{D} \rightarrow \bar{H}_{D}(\overline{\mathbf{4}}) \oplus \bar{S}_{D}(\mathbf{1}), \\
& \Sigma_{D} \rightarrow \Xi(\mathbf{1 5}) \oplus h_{D}^{\prime}(\overline{\mathbf{4}}) \oplus \bar{h}_{D}^{\prime}(\overline{\mathbf{4}}) \oplus S_{D}^{\prime}(\mathbf{1}) .
\end{aligned}
$$

Below $M_{\text {DGUT }}, S U(4)_{\text {DGUT }}$ is broken down to $S U(3)_{D} \times$ $U(1)_{D}$. We assume a pair of $U(1)_{D}$ charged Higgs multiplets remains almost massless while all the other components in Eqs. (7) and (8) obtain masses of $\mathcal{O}\left(M_{\mathrm{DGUT}}\right)$. The $U(1)_{D}$ charged Higgs multiplet breaks the $U(1)_{D}$ symmetry at the low-energy scale.

Since $\left(S_{D}, \bar{S}_{D}\right)$ do not obtain the VEVs, the matter fields in the dark sector do not obtain masses from the Yukawa interactions in Eq. (1). To generate the mass term, we assume interactions to $X_{D}$ 's such as

$W=y_{u} \Psi_{D} \Psi_{D} X_{D}+y_{d} \Psi_{D} \Phi_{D} \bar{X}_{D}+\frac{y_{e}^{\prime}}{M_{\mathrm{Pl}}} \Psi_{D} \Sigma_{D} \Phi_{D} \bar{X}_{D}$,

with tiny coupling constants. ${ }^{3}$ In the following, we take the masses of the dark quarks to be free parameters. For a successful model of ADM, the dynamical scale of $S U(3)_{D}$, $\Lambda_{\mathrm{QCD}}$, should be of $\mathcal{O}(1) \mathrm{GeV}$. At least one generation of the quarks should be lighter than $\Lambda_{\mathrm{QCD}}$, so that the lightest dark baryon can be the DM. ${ }^{4}$ The last term in Eq. (9) split the masses of the dark quarks and leptons in $A_{D}, Q_{D}$, and

\footnotetext{
${ }^{2}$ The mass of the $S U(4)_{\text {DGUT }}$ singlet component of $\left(X_{D}, \bar{X}_{D}\right)$ is of $\mathcal{O}\left(M_{\text {DGUT }}\right)$.

${ }^{3}$ The corresponding operators in the visible sector do not affect the phenomenology of the SSM.

${ }^{4}$ The dark baryon self-interactions mediated by the dark mesons may also realize the velocity-dependent cross section, and its implication for structure formation is worth investigating (see Ref. [89] for a review).
} 
$\bar{Q}_{D}$. We assume that the lightest dark lepton is heavier than

$\Lambda_{\mathrm{QCD}^{\prime}}$ so that the rapid dark matter decay is avoided [73].

The visible and dark sectors are connected through superpotential $W_{N}$ of the right-handed neutrinos,

$$
\begin{aligned}
W_{N}= & \Phi_{V} y_{N} \bar{N} H_{V}+\Phi_{D} y_{N} \bar{N}^{\prime} H_{D} \\
& +\Phi_{V} Y_{N} \bar{N}^{\prime} H_{V}+\Phi_{D} Y_{N} \bar{N} H_{D} \\
& + \text { (mass terms) },
\end{aligned}
$$

where $y_{N}$ and $Y_{N}$ are Yukawa coupling constants. The mass terms of the right-handed neutrinos (denoted by $M_{R}$ collectively) softly break $U(1)_{X}$. The global $U(1)_{X}$ symmetry renders $M_{R}$ much smaller than the GUT scale technically natural since $U(1)_{X}$ is restored in the limit of $M_{R} \rightarrow 0$. Couplings of $\bar{N}$ to $\Phi_{V}$ realize thermal leptogenesis and tiny neutrino masses via the type-I seesaw mechanism [90-94], while the couplings of $\bar{N}^{\prime}$ are irrelevant because we assume that $\bar{N}^{\prime}$ is much heavier than $\bar{N}$.

The dark neutrinos (included in $\Phi_{D}$ 's) can easily have either Majorana or Dirac mass terms of $\mathcal{O}\left(M_{\text {DGUT }}\right)$, and thus our framework is consistent with cosmological constraints on light particles. For example, the Majorana mass would be generated from $U(1)_{X}$ breaking higherdimensional operators such as $\left(X_{D} \Phi_{D}\right)^{2}$, while the Dirac mass would be generated from the usual Yukawa coupling, $X_{D} \Phi_{D} \bar{N}^{\prime}$.

As shown in Ref. [73], the $B-L$ portal operators between the two sectors are generated by integrating out the right-handed neutrino and the dark-colored Higgs boson,

$$
W_{\text {eff. }}=\frac{\left(Y_{d}\right)_{i j}\left(Y_{N}\right)_{k l}}{\sqrt{2} M_{C}} \epsilon_{a b c}\left(\bar{U}_{i}^{\prime a} \bar{D}_{j}^{\prime b}\right)\left(\bar{D}_{k}^{\prime c} \bar{N}_{l}\right)
$$

Here, $\bar{U}^{\prime}$ and $\bar{D}^{\prime}$ denote the dark quark superfields, $\epsilon_{a b c}$ is the totally antisymmetric tensor of $S U(3)_{D}$, and $M_{C}$ is the mass of the dark-colored Higgs. Below the energy scale of $M_{R}$, one obtains

$$
W_{\text {eff. }}=-\frac{y_{N}\left(Y_{d}\right)_{i j}\left(Y_{N}\right)_{k l}}{M_{C} M_{R}} \epsilon_{a b c} \bar{U}_{i}^{\prime a} \bar{D}_{j}^{\prime b} \bar{D}_{k}^{\prime c}\left(L_{l} H_{u}\right),
$$

where $L$ and $H_{u}$ denote lepton and Higgs superfields in the SSM sector, respectively. The decoupling temperature of these portal interactions should be below $M_{R}$; otherwise these interactions decouple before the $B-L$ asymmetry is generated. Meanwhile, the consistency of renormalizable operators requires $M_{R}<M_{C}$. These portal interactions successfully mediate the $B-L$ asymmetry generated by thermal leptogenesis for $M_{R}<M_{C} \lesssim 10^{2} Y_{N} Y_{d} M_{R}$ [73]. Here, we use a seesaw relation of the neutrino Yukawa coupling $y_{N}^{2} v^{2}=m_{\nu} M_{R}, m_{\nu} \simeq 0.1 \mathrm{eV}$, and the SM Higgs VEV $v$. For the split supersymmetry with the sfermion masses in $\mathcal{O}(100) \mathrm{TeV}$, the ratio of the VEVs of two Higgs doublets, $\tan \beta$, is required to be $\tan \beta \simeq 2$ to reproduce the observed Higgs boson mass. In this case, the largest downtype Yukawa coupling is of $\mathcal{O}\left(10^{-2}\right)$, and hence, a rather large $Y_{N}$ is required.

It should be noted that the above portal interactions require at least two generations of dark quarks to be nonvanishing. In the following, we leave only the two generations of $U^{\prime}$ and $D^{\prime}$ below the $M_{\text {DGUT }}$ scale, for simplicity.

In Fig. 1, we show the one-loop running of the gauge couplings in the two sectors. We take $M_{\text {DGUT }}=$ $8 \times 10^{10} \mathrm{GeV}$ and the corresponding $S U(5)_{\text {DGUT }}$ breaking scale at $10^{14} \mathrm{GeV}$ as an example. In this plot, the dark confinement scale $\Lambda_{\mathrm{QCD}}$, where $S U(3)_{D}$ coupling $\alpha_{s}^{\prime-1}\left(\Lambda_{\mathrm{QCD}^{\prime}}\right)$ vanishes, is about $2.8 \mathrm{GeV}$, which is consistent with the dark baryon mass $m_{\mathrm{DM}}=O(1) \mathrm{GeV}$ determined by the asymmetries in two sectors $[50,95,96]$. Therefore, the dark baryons with the mass of $\mathcal{O}(1) \mathrm{GeV}$ can be naturally realized as a consequence of the $\mathbb{Z}_{2}$ symmetry at the high-energy scale.

\section{Baryon-DM coincidence}

The dark confinement scale is restricted in our model since the unified couplings in the two sectors are identified at the GUT scale. The analytic solution of renormalization

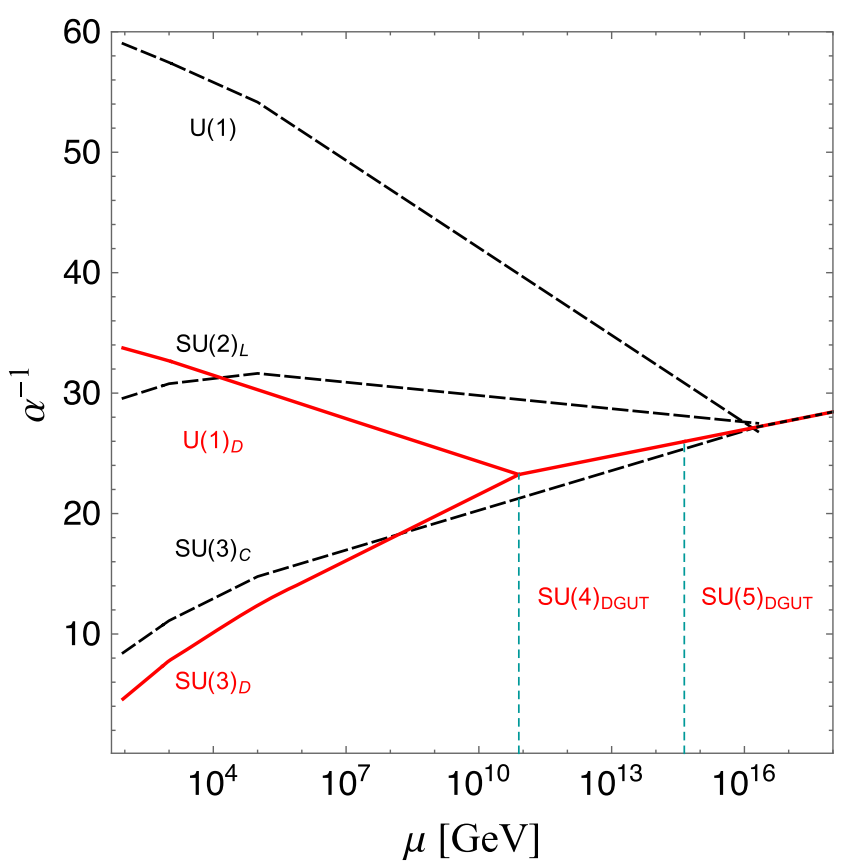

FIG. 1. Renormalization scale $\mu$ dependence of the gauge couplings in two sectors. The broken lines show the (S)SM gauge couplings, while the red lines show the running of dark gauge couplings. We take a split spectrum for sparticles: the gaugino scale is $1 \mathrm{TeV}$ and the sfermion scale is $100 \mathrm{TeV}$ as reference values. We also assume that $M_{\text {DGUT }}=8 \times 10^{10} \mathrm{GeV}$ where $S U(3)_{D} \times U(1)_{D}$ unifies into $S U(4)_{\text {DGUT }}$. 
group equations for gauge couplings gives the dark confinement scale,

$\Lambda_{\mathrm{QCD}^{\prime}} \simeq 2.8 \mathrm{GeV}\left(\frac{M_{\mathrm{SUSY}}}{10^{2} \mathrm{TeV}}\right)^{\frac{4}{25}}\left(\frac{M_{\mathrm{DGUT}}}{8 \times 10^{10} \mathrm{GeV}}\right)^{\frac{9}{25}}$,

where $M_{\text {SUSY }}$ is a typical mass scale of (dark) sfermions for two-generation matter in the dark sector below $M_{\text {DGUT }}$.

Figure 2 shows the ratio of the confinement scales in the two sectors. The right axis of Fig. 2 shows the ratio of energy densities of ADM and baryon, which is obtained from that of the confinement scales by multiplying the ratio of asymmetries stored in two sectors when the two generations of $U^{\prime}$ and $D^{\prime}$ are assumed [96]. Here we take $S U(5)_{\text {DGUT }}$ breaking scale smaller than $M_{\mathrm{VGUT}}$. As reference values, we take the gauginos and Higgsinos to be about $1 \mathrm{TeV}$ and the sfermion masses to be $M_{\mathrm{SUSY}}=$ $10^{2} \mathrm{TeV}\left(10^{3} \mathrm{TeV}\right)$ on the red (orange) line. We note that the confinement scales do not change significantly even if the Higgsinos in two sectors are lighter than $1 \mathrm{TeV}$. As a prominent feature of the model, the dark confinement scale is no longer a free parameter in our scenario and is predicted to be in the range of $\mathcal{O}\left(1-10^{2}\right) \Lambda_{\mathrm{QCD}}$ for a wide range of $M_{\mathrm{DGUT}} / M_{\mathrm{VGUT}}$. Here we take $\Lambda_{\mathrm{QCD}} \simeq 0.3 \mathrm{GeV}$. This shows that the $\mathbb{Z}_{2}$ symmetry successfully predicts that the dynamical scales are close to each other, despite the vacuum structures being completely different between two sectors below the $M_{\mathrm{VGUT}}$ scale.

It should also be noted that the kinetic mixing parameter is predicted to be $\epsilon \simeq 10^{-10}-10^{-8}$ for $\Lambda_{\mathrm{QCD}^{\prime}} / \Lambda_{\mathrm{QCD}} \simeq 5-50$. This feature is another advantage of the present model.

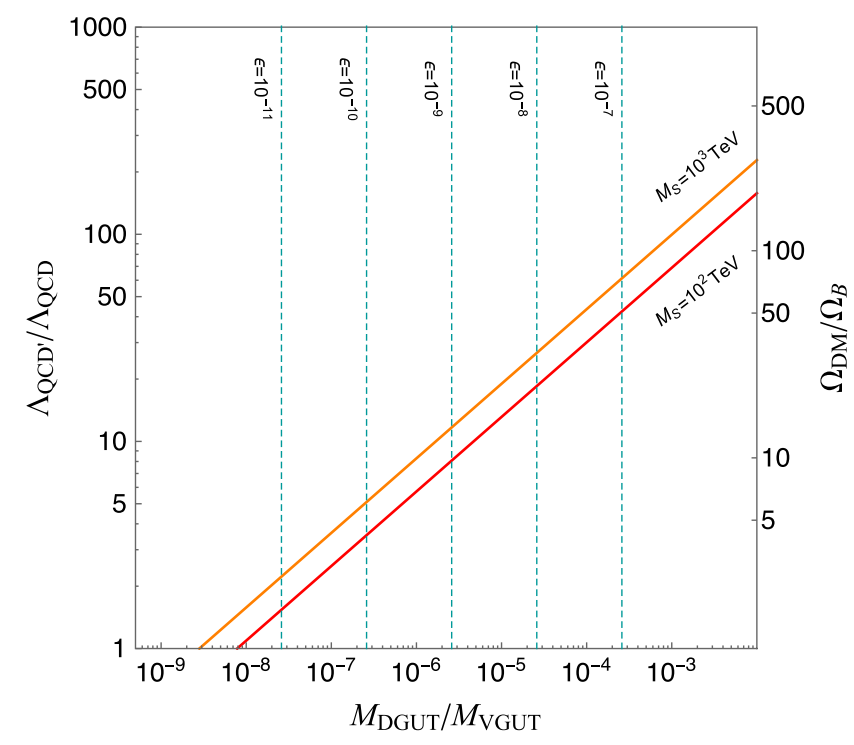

FIG. 2. Confinement scale and energy density as a function of $M_{\mathrm{DGUT}} / M_{\mathrm{VGUT}}$. Green-dashed lines represent the magnitude of the kinetic mixing parameter $\epsilon$ given in Eq. (4) with $c_{\epsilon}=1$.
For the present model, $M_{\text {DGUT }}$ is bounded from below. The lower dark GUT scale, $M_{\text {DGUT }} \lesssim 10^{9} \mathrm{GeV}$, would also be incompatible with the standard thermal leptogenesis [74] (see Refs. [75-77] for reviews), since the standard thermal leptogenesis requires the high reheating temperature of $T_{R}>M_{R}>10^{9} \mathrm{GeV}$ and meanwhile our framework requires $M_{R}<M_{C} \sim M_{\text {DGUT }}{ }^{5}$ The lower dark GUT scale, $M_{\text {DGUT }} \lesssim 10^{9} \mathrm{GeV}$, would also be confronted with rapid dark matter decay through the portal interactions [96]. Once the form factor of the DM is determined in naive dimensional analysis, its lifetime is approximately given by

$$
\begin{aligned}
\tau_{\mathrm{DM}} & \simeq\left(\frac{m_{\nu} m_{\mathrm{DM}}^{5}}{M_{C}^{2} M_{R} M_{\mathrm{SUSY}}^{2}}\right)^{-1}\left(\frac{\alpha}{4 \pi}\right)^{-2} \\
& \simeq 10^{14} \text { [years] } \frac{M_{C}^{2} M_{R}}{\left(10^{9} \mathrm{GeV}\right)^{3}}\left(\frac{M_{\mathrm{SUSY}}}{10^{2} \mathrm{TeV}}\right)^{2} .
\end{aligned}
$$

Here, we use a seesaw relation of neutrino Yukawa coupling $y_{N}^{2} v^{2}=m_{\nu} M_{R}$, again. Since Eq. (12) provides the portal interaction with sfermions, the dark matter decay operators arise with a loop factor as with nucleon decay in SUSY GUTs. $M_{\text {SUSY }}$ is a typical mass scale of (dark) sfermions. We assume the loop factor to be $\alpha / 4 \pi \simeq 0.01$.

\section{CONCLUDING REMARKS}

In this work, we have proposed the mirrored GUT framework in which the baryon-DM coincidence is naturally explained. The framework relates the masses of baryon and DM (dynamical scales) and also the number (asymmetry) densities.

In contrast to the models keeping mirror symmetry at a low-energy scale, it is interesting that our framework leads to rich phenomenology and testable signatures [50]. DM decays into SM neutrinos through the intermediate-energy portal interactions $[96,97]$. DM annihilates through a dark neutron-antineutron oscillation [98]. When DM is composed of dark charged baryons, DM interacts with the SM fermions through tiny kinetic mixing between photon and dark photon. The monopoles from the $S U(4)_{\text {DGUT }} \rightarrow$ $S U(3)_{D} \times U(1)_{D}$ breaking are finally confined by the cosmic string after the $U(1)_{D}$ breaking, and then the monopole density decreases efficiently by following the scaling relation of the cosmic string $[99,100]$. [Here, the maximum temperature of the Universe after inflation is higher than the $S U(4)_{\text {DGUT }}$ breaking scale.] It is worth investigating the monopoles and their annihilation, but it is beyond the scope of this work to discuss it in detail. A first

\footnotetext{
${ }^{5}$ The high reheating temperature causes the overabundance of LSPs from gravitino decay when the visible Higgsino is lighter than the dark Higgsino. In that case, the reheating temperature should be less than $10^{9}-10^{10} \mathrm{GeV}$. This constraint puts an upper bound on $M_{C} \sim M_{\text {DGUT }} \lesssim 10^{2} Y_{N} Y_{d} M_{R}$ in the present model.
} 
order phase transition of the dark QCD would be a probe of our framework [101].

We regard the specific model in this paper as a proof of concept and have not addressed the origins of several finetuned parameters. Fine-tunings of parameters are just technically natural thanks to SUSY and furthermore most tuned parameters are irrelevant to explain the baryon-DM coincidence puzzle. However, it is to be addressed in the future why chiral symmetry breaking in the dark sector is so tiny, although the dark sector is a vectorlike theory below the $S U(5)_{\text {DGUT }} \rightarrow S U(4)_{\text {DGUT }}$ breaking. We may consider a variant of the present model to ameliorate the parameter tunings in the superpotentials (for example, introducing chiral symmetry to suppress the Higgs $\mu$-term). Although our present model is not fully satisfactory, it demonstrates a new vast field of the DM-model building to be explored.

\section{ACKNOWLEDGMENTS}

A. K. acknowledges the Mainz Institute for Theoretical Physics (MITP) of the Cluster of Excellence PRISMA+ (Grant No. 39083149) for enabling A. K. to complete a significant portion of this work. The work of A. K. and T. K. is supported by IBS under Grant No. IBS-R018-D1. This work is also supported by JSPS KAKENHI Grants No. 15H05889, No. 16H03991, No. 17H02878, and No. $18 \mathrm{H} 05542$ (M. I.) and by World Premier International Research Center Initiative (WPI Initiative), MEXT, Japan.
[1] N. Aghanim et al. (Planck Collaboration), arXiv: 1807.06209.

[2] I. Yu. Kobzarev, L. B. Okun, and I. Ya. Pomeranchuk, Yad. Fiz. 3, 1154 (1966) [Sov. J. Nucl. Phys. 3, 837 (1966)].

[3] S. I. Blinnikov and M. Khlopov, Astron. Zh. 60, 632 (1983) [Sov. Astron. 27, 371 (1983)].

[4] E. W. Kolb, D. Seckel, and M. S. Turner, Nature (London) 314, 415 (1985).

[5] M. Yu. Khlopov, G. M. Beskin, N. E. Bochkarev, L. A. Pustylnik, and S. A. Pustylnik, Astron. Zh. 68, 42 (1991) [Sov. Astron. 35, 21 (1991)].

[6] H. M. Hodges, Phys. Rev. D 47, 456 (1993).

[7] R. Foot, Int. J. Mod. Phys. D 13, 2161 (2004).

[8] Z. Chacko, H.-S. Goh, and R. Harnik, Phys. Rev. Lett. 96, 231802 (2006).

[9] N. Craig and A. Katz, J. Cosmol. Astropart. Phys. 10 (2015) 054.

[10] I. G. Garcia, R. Lasenby, and J. March-Russell, Phys. Rev. Lett. 115, 121801 (2015).

[11] M. Farina, J. Cosmol. Astropart. Phys. 11 (2015) 017.

[12] R. Barbieri, L. J. Hall, and K. Harigaya, J. High Energy Phys. 11 (2016) 172.

[13] S. J. Lonsdale and R. R. Volkas, Phys. Rev. D 90, 083501 (2014); 91, 129906(E) (2015).

[14] P.-H. Gu, J. Cosmol. Astropart. Phys. 12 (2014) 046.

[15] S. B. Gudnason, C. Kouvaris, and F. Sannino, Phys. Rev. D 74, 095008 (2006).

[16] D. D. Dietrich and F. Sannino, Phys. Rev. D 75, 085018 (2007).

[17] M. Y. Khlopov and C. Kouvaris, Phys. Rev. D 77, 065002 (2008).

[18] M. Y. Khlopov and C. Kouvaris, Phys. Rev. D 78, 065040 (2008).

[19] R. Foadi, M. T. Frandsen, and F. Sannino, Phys. Rev. D 80, 037702 (2009).

[20] J. Mardon, Y. Nomura, and J. Thaler, Phys. Rev. D 80, 035013 (2009).
[21] G. D. Kribs, T. S. Roy, J. Terning, and K. M. Zurek, Phys. Rev. D 81, 095001 (2010).

[22] R. Barbieri, S. Rychkov, and R. Torre, Phys. Lett. B 688 , 212 (2010).

[23] M. Blennow, B. Dasgupta, E. Fernandez-Martinez, and N. Rius, J. High Energy Phys. 03 (2011) 014.

[24] R. Lewis, C. Pica, and F. Sannino, Phys. Rev. D 85, 014504 (2012).

[25] T. Appelquist et al. (Lattice Strong Dynamics), Phys. Rev. D 88, 014502 (2013).

[26] A. Hietanen, R. Lewis, C. Pica, and F. Sannino, J. High Energy Phys. 12 (2014) 130.

[27] J. M. Cline, Z. Liu, G. D. Moore, and W. Xue, Phys. Rev. D 90, 015023 (2014).

[28] T. Appelquist et al. (Lattice Strong Dynamics), Phys. Rev. D 89, 094508 (2014).

[29] A. Hietanen, R. Lewis, C. Pica, and F. Sannino, J. High Energy Phys. 07 (2014) 116.

[30] G. Krnjaic and K. Sigurdson, Phys. Lett. B 751, 464 (2015).

[31] W. Detmold, M. McCullough, and A. Pochinsky, Phys. Rev. D 90, 115013 (2014).

[32] W. Detmold, M. McCullough, and A. Pochinsky, Phys. Rev. D 90, 114506 (2014).

[33] M. Asano and R. Kitano, J. High Energy Phys. 09 (2014) 171.

[34] J. Brod, J. Drobnak, A. L. Kagan, E. Stamou, and J. Zupan, Phys. Rev. D 91, 095009 (2015).

[35] O. Antipin, M. Redi, and A. Strumia, J. High Energy Phys. 01 (2015) 157.

[36] E. Hardy, R. Lasenby, J. March-Russell, and S. M. West, J. High Energy Phys. 06 (2015) 011.

[37] T. Appelquist et al., Phys. Rev. D 92, 075030 (2015).

[38] T. Appelquist et al., Phys. Rev. Lett. 115, 171803 (2015).

[39] O. Antipin, M. Redi, A. Strumia, and E. Vigiani, J. High Energy Phys. 07 (2015) 039. 
[40] E. Hardy, R. Lasenby, J. March-Russell, and S. M. West, J. High Energy Phys. 07 (2015) 133.

[41] R. T. Co, K. Harigaya, and Y. Nomura, Phys. Rev. Lett. 118, 101801 (2017).

[42] K. R. Dienes, F. Huang, S. Su, and B. Thomas, Phys. Rev. D 95, 043526 (2017).

[43] H. Ishida, S. Matsuzaki, and Y. Yamaguchi, Prog. Theor. Exp. Phys. 2017, 103B01 (2017).

[44] S. J. Lonsdale, M. Schroor, and R. R. Volkas, Phys. Rev. D 96, 055027 (2017).

[45] J. M. Berryman, A. de Gouvêa, K. J. Kelly, and Y. Zhang, Phys. Rev. D 96, 075010 (2017).

[46] M. I. Gresham, H. K. Lou, and K. M. Zurek, Phys. Rev. D 96, 096012 (2017).

[47] M. I. Gresham, H. K. Lou, and K. M. Zurek, Phys. Rev. D 97, 036003 (2018).

[48] A. Mitridate, M. Redi, J. Smirnov, and A. Strumia, J. High Energy Phys. 10 (2017) 210.

[49] M. I. Gresham, H. K. Lou, and K. M. Zurek, Phys. Rev. D 98, 096001 (2018).

[50] M. Ibe, A. Kamada, S. Kobayashi, and W. Nakano, J. High Energy Phys. 11 (2018) 203.

[51] E. Braaten, D. Kang, and R. Laha, J. High Energy Phys. 11 (2018) 084.

[52] A. Francis, R. J. Hudspith, R. Lewis, and S. Tulin, J. High Energy Phys. 12 (2018) 118.

[53] Y. Bai, A. J. Long, and S. Lu, Phys. Rev. D 99, 055047 (2019).

[54] X. Chu, C. Garcia-Cely, and H. Murayama, arXiv: 1901.00075.

[55] G. D. Kribs and E. T. Neil, Int. J. Mod. Phys. A 31, 1643004 (2016).

[56] S. Nussinov, Phys. Lett. 165B, 55 (1985).

[57] S. M. Barr, R. S. Chivukula, and E. Farhi, Phys. Lett. B 241, 387 (1990).

[58] S. M. Barr, Phys. Rev. D 44, 3062 (1991).

[59] D. B. Kaplan, Phys. Rev. Lett. 68, 741 (1992).

[60] S. Dodelson, B. R. Greene, and L. M. Widrow, Nucl. Phys. B372, 467 (1992).

[61] V. A. Kuzmin, Phys. Part. Nucl. 29, 257 (1998); Phys. At. Nucl. 61, 1107 (1998).

[62] M. Fujii and T. Yanagida, Phys. Lett. B 542, 80 (2002).

[63] R. Kitano and I. Low, Phys. Rev. D 71, 023510 (2005).

[64] S. B. Gudnason, C. Kouvaris, and F. Sannino, Phys. Rev. D 73, 115003 (2006).

[65] R. Kitano, H. Murayama, and M. Ratz, Phys. Lett. B 669 , 145 (2008).

[66] D. E. Kaplan, M. A. Luty, and K. M. Zurek, Phys. Rev. D 79, 115016 (2009).

[67] H. Davoudiasl and R. N. Mohapatra, New J. Phys. 14, 095011 (2012).

[68] K. Petraki and R. R. Volkas, Int. J. Mod. Phys. A 28, 1330028 (2013).

[69] K. M. Zurek, Phys. Rep. 537, 91 (2014).

[70] D. S. M. Alves, S. R. Behbahani, P. Schuster, and J. G. Wacker, Phys. Lett. B 692, 323 (2010).
[71] D. Spier Moreira Alves, S. R. Behbahani, P. Schuster, and J. G. Wacker, J. High Energy Phys. 06 (2010) 113.

[72] M. Blennow, E. Fernandez-Martinez, O. Mena, J. Redondo, and P. Serra, J. Cosmol. Astropart. Phys. 07 (2012) 022.

[73] M. Ibe, A. Kamada, S. Kobayashi, T. Kuwahara, and W. Nakano, J. High Energy Phys. 03 (2019) 173.

[74] M. Fukugita and T. Yanagida, Phys. Lett. B 174, 45 (1986).

[75] G. F. Giudice, A. Notari, M. Raidal, A. Riotto, and A. Strumia, Nucl. Phys. B685, 89 (2004).

[76] W. Buchmüller, R. D. Peccei, and T. Yanagida, Annu. Rev. Nucl. Part. Sci. 55, 311 (2005).

[77] S. Davidson, E. Nardi, and Y. Nir, Phys. Rep. 466, 105 (2008).

[78] T. Goto and T. Nihei, Phys. Rev. D 59, 115009 (1999).

[79] H. Murayama and A. Pierce, Phys. Rev. D 65, 055009 (2002).

[80] K. Abe et al. (Super-Kamiokande Collaboration), Phys. Rev. D 90, 072005 (2014).

[81] V. Takhistov (Super-Kamiokande Collaboration), in Proceedings of the 51st Rencontres de Moriond on Electroweak Interactions and Unified Theories: La Thuile, Italy, 2016 (2016), pp. 437-444.

[82] N. Arkani-Hamed and S. Dimopoulos, J. High Energy Phys. 06 (2005) 073.

[83] G. F. Giudice and A. Romanino, Nucl. Phys. B699, 65 (2004); B706, 487(E) (2005).

[84] J. D. Wells, Phys. Rev. D 71, 015013 (2005).

[85] M. Ibe, T. Moroi, and T. T. Yanagida, Phys. Lett. B 644, 355 (2007).

[86] M. Bauer, P. Foldenauer, and J. Jaeckel, J. High Energy Phys. 07 (2018) 094.

[87] J. H. Chang, R. Essig, and S. D. McDermott, J. High Energy Phys. 01 (2017) 107.

[88] J. H. Chang, R. Essig, and S. D. McDermott, J. High Energy Phys. 09 (2018) 051.

[89] S. Tulin and H.-B. Yu, Phys. Rep. 730, 1 (2018).

[90] P. Minkowski, Phys. Lett. 67B, 421 (1977).

[91] T. Yanagida, Conf. Proc. C 7902131, 95 (1979).

[92] S. L. Glashow, NATO Sci. Ser. B 61, 687 (1980).

[93] M. Gell-Mann, P. Ramond, and R. Slansky, Conf. Proc. C 790927, 315 (1979).

[94] R. N. Mohapatra and G. Senjanović, Phys. Rev. Lett. 44, 912 (1980).

[95] M. Ibe, S. Matsumoto, and T. T. Yanagida, Phys. Lett. B 708, 112 (2012).

[96] H. Fukuda, S. Matsumoto, and S. Mukhopadhyay, Phys. Rev. D 92, 013008 (2015).

[97] B. Feldstein and A. L. Fitzpatrick, J. Cosmol. Astropart. Phys. 09 (2010) 005.

[98] M. R. Buckley and S. Profumo, Phys. Rev. Lett. 108, 011301 (2012).

[99] M. Hindmarsh, K. Rummukainen, and D. J. Weir, Phys. Rev. D 95, 063520 (2017).

[100] M. Hindmarsh, A. Kormu, A. Lopez-Eiguren, and D. J. Weir, Phys. Rev. D 98, 103533 (2018).

[101] P. Schwaller, Phys. Rev. Lett. 115, 181101 (2015). 